\title{
PROGRESS IN TUNNEL SENSORS
}

\author{
J. Grade, A. Barzilai, J.K. Reynolds, C.H. Liu, A. Partridge, and T.W. Kenny \\ Department of Mechanical Engineering, Terman 551 \\ Stanford University, Stanford, CA 94305-4021
}

\author{
T.R. VanZandt, L.M. Miller, and J.A. Podosek \\ Jet Propulsion Laboratory, Pasadena, CA 91109
}

\begin{abstract}
For several years, research has been underway on the use of electron tunneling as a displacement transducer in microsensors. Several prototype devices have been developed which illustrate the potential advantages of tunneling transducers. In this report, we focus on recent measurements of noise and drift which indicate that tunneling transducers can perform very well over long periods. In addition, we report on recent tunnel sensor development efforts, including a MCNC-based test device, an integrated controller for tunneling transducers, and a high-performance tunneling seismometer based on a modified geophone.
\end{abstract}

\section{INTRODUCTION}

Silicon micromachining has enabled the miniaturization of nearly every displacement transducer-based sensor. In many cases, the miniaturization of these devices has come with a reduction in sensitivity. The reduced sensitivity is due to two effects. The first effect is a reduction in the coupling of the external signal to the displacement of a sensor component. A miniaturized pressure sensor, for example, undergoes a smaller deflection because the area of the diaphragm has been reduced. The second effect is a reduction in the performance of the displacement transducer with the size of the device. A capacitive pressure sensor, for example, becomes less sensitive as the area of the capacitive electrodes is reduced while miniaturizing the device.

Electron tunneling has been studied as an important displacement transducer for microsensors because of its high sensitivity and scale independence. In tunneling, a nA current is measured, and $1 \%$ fluctuations in this current correspond to displacements of less than $0.1 \AA$. This sensitivity is independent of the lateral dimensions of the electrodes, since tunneling only requires 1 metal atom on the surface of each side of the gap.

In the last 2 years alone, published work on tunneling sensors has included an infrared detector[1], several accelerometers[2-4], a pressure sensor[5], and a magnetometer[6]. All of this work serves to illustrate the breadth of applications for the tunneling transducer and the variety of possible fabrication techniques which may be used to build them. A complete description of the issues to be considered during design, fabrication, and operation of micromachined tunneling transducers has been published.[7]

Despite this growing research effort, there is still broad concern about the utility of tunneling sensors for real applications. Generally, concerns are expressed about the fabrication processes necessary to produce appropriate characteristics, the stability of tunneling transducers, and the packaging requirements necessary to preserve sensor performance. In this report, we will review recent work directed towards these issues, and describe several development efforts which serve to illustrate the simplicity, applicability and performance of tunneling transducers.

\section{LOW-FREQUENCY NOISE MEASUREMENTS}

The properties of tunneling transducers are known to rely on the location of atoms on a pair of electrodes. If the atoms rearrange themselves, the current between the electrodes will fluctuate, leading to errors in the measurement of signals applied to the device. This dependence on the position of individual atoms is unique to tunneling; capacitive transducers are expected to be more stable because they are only sensitive to the average separation between extremely large numbers of atoms. Gold surfaces are favored for tunneling electrodes because gold resists chemical reaction with the constituents of most potential operating environments. Unfortunately, gold is a soft metal, and gold surface atoms are known to re-arrange at regular intervals at room temperature. Arrangements of atoms which feature extremely sharp tip-like structures can be expected to be particularly unstable because energy considerations favor flat (111) crystal surfaces.

In addition to gold atom rearrangements, errors can be expected due to the migration of physically-adsorbed molecules, such as $\mathrm{H}_{2} \mathrm{O}$, through the tunneling region. Other possible sources of error include variations in the mechanical characteristics of the structure supporting the electrodes, and variations in the electrical properties of the feedback circuit elements.

Since we are interested in the fundamental performance of tunneling transducers, we need to consider how these various sources of noise can contribute to significant errors in position. For example, the migration of adsorbates through the tunneling region should only be important at high frequencies because this mechanism cannot produce an accumulation of error. Changes in the structure due to thermal expansion should only be important at low frequency, because the temperature cannot change quickly. To understand the phenomena which limit the performance of tunneling sensors, we have focused on noise measurements.

An example of a micromachined device which uses tunneling currents to detect small forces is the membrane transducer, shown in Fig. 1. This device operates by electrostatically deflecting the membrane down towards the tip. When a tunneling current of order $1 \mathrm{nA}$ appears between the tip and the membrane, a feedback voltage is added to the voltage on the deflection electrode so as to maintain the current at a constant value. If an external force is applied to the membrane, the feedback circuit responds by applying a balancing electrostatic force. By monitoring the feedback control voltage, it is possible to determine the external forces. 


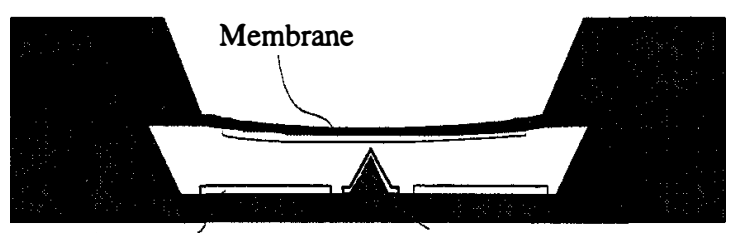

Deflection Electrodes

Tip

Fig. 1 This drawing shows the design of the membrane transducer. In this design, the gap between deflection electrode and membrane is about $40 \mu \mathrm{m}$, and the tip is about $40 \mu \mathrm{m}$ tall. The membrane is $2 \mathrm{~mm} \times 2 \mathrm{~mm} \times 0.5 \mu \mathrm{m}$.

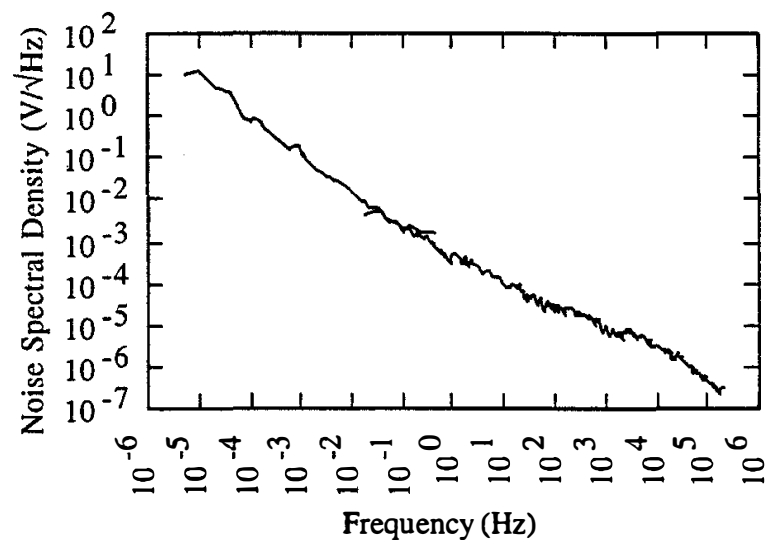

Fig. 2 This graph shows the measured noise spectral density for membrane tunneling transducers over a very broad range of frequencies.

A complete fabrication process and experimental characterization of this membrane transducer has been presented elsewhere. [7] In summary, this device features a control bandwidth in excess of $50 \mathrm{kHz}$, a force resolution of $10^{-11} \mathrm{~N} / \mathrm{VHz}$, and a very simple fabrication and assembly technique. Devices of this type have been operated continuously for more than 3 years without failure. This device is the basis of the successful tunneling infrared sensor and magnetometer, and has been studied extensively at JPL and elsewhere.

All of the tunnel sensor development projects carried out in the JPL and Stanford groups have been constrained to applications which are within the known performance capabilities of tunneling transducers. One component of this constraint has been the known noise spectra of tunneling transducers. Figure 2 shows a measurement of the noise spectral density of the control voltage for membrane-style sensors over a very broad frequency range. This measurement has been repeated many times for many sensors in several locations, and is characterized by a $1 / \sqrt{ } F$ shape. The sources for this noise were not identified, but its consistency persuaded us to avoid applications which would require improvement in this noise performance. The displacement sensitivity of these devices is generally around $10 \mathrm{mV} / \AA$, so the resolution at $1 \mathrm{~Hz}$ is $0.02 \AA \sqrt{ } \mathrm{Hz}$.

Several months ago, our group began logging data on the long-term drift in tunneling transducers. The purpose of those measurements was to look for an upper limit to the $1 / \sqrt{ } \mathrm{F}$ noise characteristic, because there are practical limits to the range of

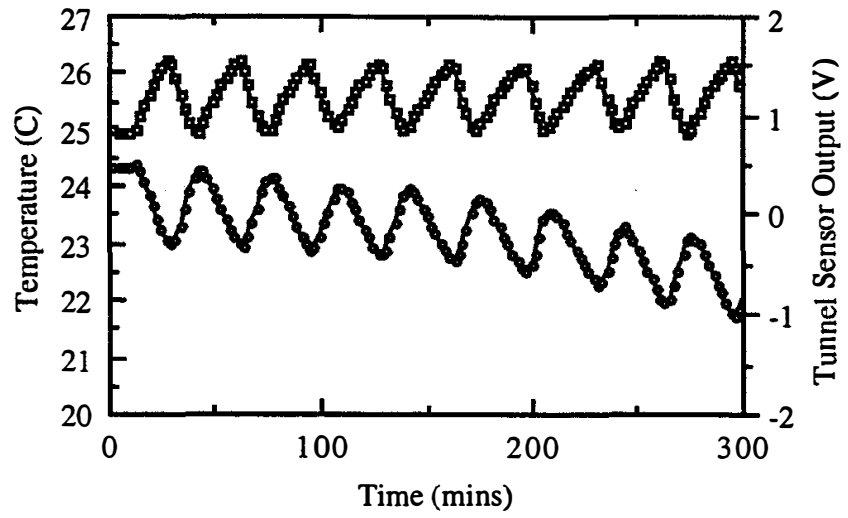

Fig. 3 This graph shows the measured output signal for a membrane transducer (lower curve), along with a measurement of the temperature near the sensor in the laboratory (upper curve)

error possible in a device of this sort. Figure 3 shows the result of an early measurement of the output signal from a membrane transducer operated at Stanford.

This initial low-frequency drift measurement is characterized by a very low-frequency oscillation. After some investigation, the source of this oscillation was identified : the laboratory is air-conditioned by a central air system which features a limit-cycle oscillation with a period of about $30 \mathrm{~min}$, depending on the heat load applied to the building. This hypothesis was confirmed by a repeat experiment which included a thermometer whose output was also logged. The result of this experiment is shown in Figure 3 along with the sensor output oscillation. Clearly, there is a strong correlation between the temperature and the tunnel sensor output.

Figure 4 is a graph of the coherence between the tunnel sensor noise spectrum and the thermometer noise spectrum. It is clear that, for frequencies below $0.1 \mathrm{~Hz}$, these signals are very well correlated (correlation $>95 \%$ ). There is one very strong conclusion which may be drawn from this measurement : More than $95 \%$ of the noise in tunneling transducers is due to temperature coefficients of the mechanical properties of the sensor or the package. If these thermal sensitivities could be eliminated, the low-frequency noise of these sensors would improve by a significant factor.

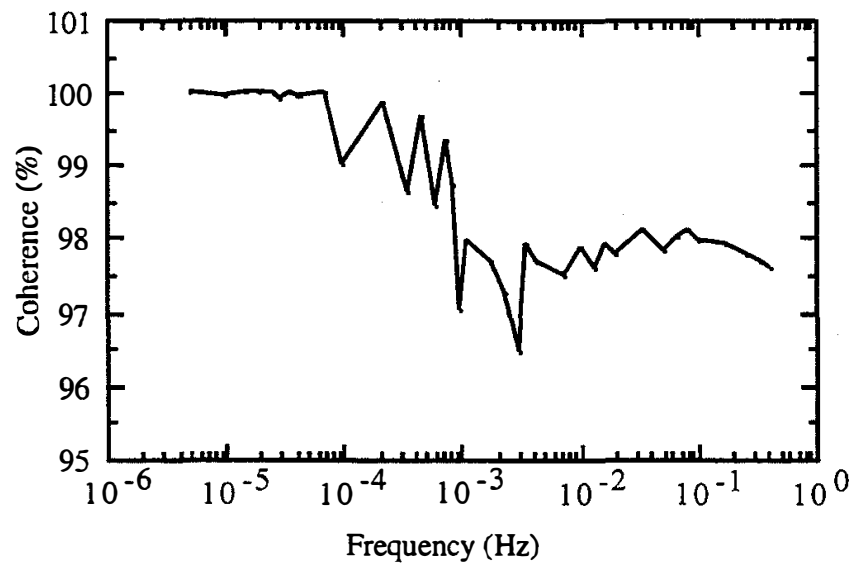

Fig. 4. This graph shows the measured coherence between the output of the membrane sensor and the thermometer. 


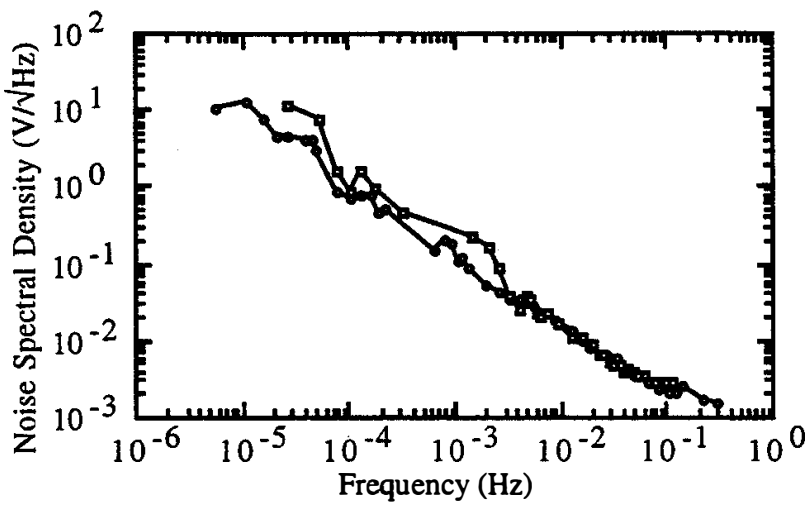

Fig. 5. This graph shows the measured Noise Spectral Density for the output signals of a sensor operated with metal spring clamps (circles) and with epoxy (squares) holding the structure together. It is clear that the replacement of clamps with epoxy has not had an important effect on the noise in this sensor. (The peaks due to air conditioner oscillation have been removed to increase clarity.)

We initially expected that this thermal sensitivity was related to the epoxy used to bond the halves of a tunneling transducer together. To test this hypothesis, we measured the noise spectrum of a tunnel sensor when its halves were clamped together with folded metal wire springs, and then when these springs were replaced with epoxy. The result is shown in Fig. 5, which indicates that the low-frequency noise in the tunneling device is not changed when the metal springs are replaced with quick-cure epoxy. This result can be explained in one of two ways : either the temperature coefficient of the stiffness of the springs and the epoxy are exactly the same, or the thermal sensitivity of the membrane sensor is dominated by some other element of the structure. We believe the latter hypothesis, and are planning a series of experiments to test this.

Figure 6 shows a plot of the noise in a membrane transducer, and a plot of the noise in the transducer which is uncorrelated with the output of our thermometer. We can clearly say that the lower curve represents the new upper bound to the fundamental noise in tunneling transducers.

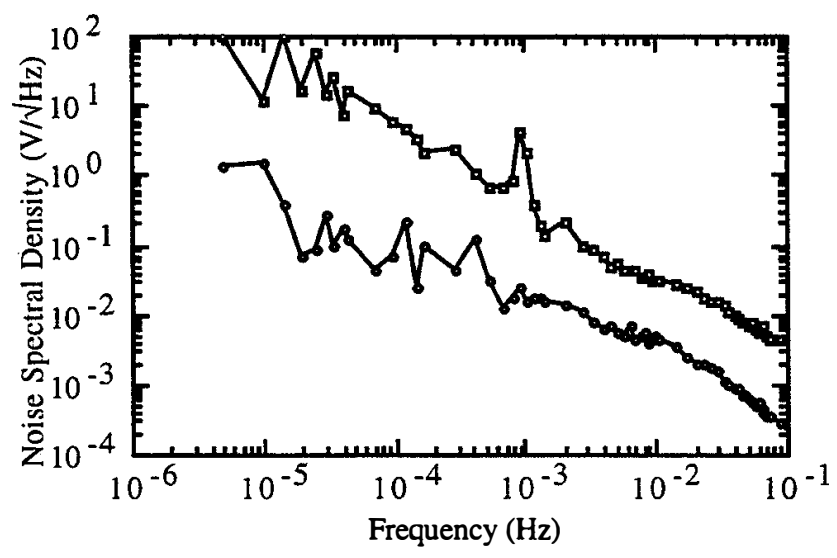

Fig. 6. This graph shows the measured noise of a typical membrane transducer(upper) and the noise which is uncorrelated with temperature measurements(lower). The lower curve represents a new upper limit to the fundamental noise in tunneling transducers of this type.
This series of measurements indicates that temperature fluctuations in a normal laboratory environment can dominate the low-frequency noise in simple tunneling structures. For our laboratory, more than $95 \%$ of the noise at all frequencies below 0.1 $\mathrm{Hz}$ is due to environmental temperature changes. Therefore, a capacitive transducer built with this mechanical structure could perform no better. It is important to note that these measurements may be limited by the accuracy of the diode thermometer that was used, and that an even greater fraction of the noise may be due to this source. The way to improve this situation is clearly by changing the mechanical design of this sensor structure. This work is currently underway.

Also, this data shows that, despite the temperature sensitivity of these membrane devices, the errors are remarkably small. These measurements allow us to be quantitative about this result. The drift in the output of a tunneling sensor may be calculated as follows :

$$
\Delta \mathrm{V}_{\mathrm{RMS}}=\sqrt{\int_{\mathrm{F} 1}^{\mathrm{F} 2}(\mathrm{NSD}(\mathrm{F}))^{2} \mathrm{dF}},
$$

where $\operatorname{NSD}(F)$ is a fit to the noise spectral density (Fig. 2) as a function of frequency between the two frequencies which represent the measurement bandwidth. Across the frequency range shown in Fig. 2, this function is reasonably well-approximated by

$$
\mathrm{NSD}(\mathrm{F})=\frac{0.5 \mathrm{mV} / \sqrt{\mathrm{Hz}}}{\sqrt{\mathrm{F} / 1 \mathrm{~Hz}}} .
$$

Suppose we were to average the output of a membrane sensor for 1 second, and repeat the measurement an hour later. The RMS average difference between the results would be approximated by taking the above integral from $\mathrm{Fl}=0.0003 \mathrm{~Hz}$ to $\mathrm{F} 2=1 \mathrm{~Hz}$. The result of this calculation is a change in output voltage of $1.5 \mathrm{mV}$, which corresponds to an average error in position of only $0.15 \AA$. This is considerably smaller than the lattice spacing between atoms in Gold, and indicates that atomic motion of the atoms involved in the tunneling process is extremely rare. Clearly, this level of stability could not be achieved if the control system was unable to prevent "crashes" between the electrodes. Remember that these membrane transducers operate with extremely wide control bandwidth $(>50 \mathrm{kHz})$, thereby preventing "crashes". It might be unreasonable to expect this level of stability from any tunneling system with a smaller control bandwidth.

These measurements also indicate the importance and value in measurements of noise in tunneling sensors. Researchers who have successfully implemented any tunneling device and are seeking to publish the results are strongly encouraged to report not just the signal measurements, but also the noise measurements. Both measurements are necessary to determine the applicability of these devices, and both measurements should be considered essential in any conclusive device characterization.

\section{A PROTOTYPE TUNNELING SEISMOMETER}

Seismology and resource exploration is currently carried out with inexpensive vibration sensors called geophones.[8] Geophones operate by detecting the velocity of a spring-supported coil through voltages generated as it moves through a magnetic field. Typical geophones have a suspended mass of $20 \mathrm{gm}$, a resonant frequency near $5 \mathrm{~Hz}$, total dimensions of order $3 \mathrm{~cm}$ 
diameter $\times 5 \mathrm{~cm}$ tall, a mass of 100-200 gm, and a cost of under $\$ 50$. The suspension within the geophone uses circular $\mathrm{BeCu}$ leaf springs and is significantly stiffer in the cross-axis directions than along the sensitive axis. Near their resonant frequency, geophones can detect acceleration as small as $10^{-9} \mathrm{~g}$ when operated with a 10 $\mathrm{Hz}$ bandwidth. As such, the geophone represents a very mature, high performance technology. It would be very difficult to micromachine such a device, because the low resonant frequency would require a very compliant flexural support and a large suspended mass. Significant reduction of the mass would degrade the performance of the sensor because thermal noise increases as $1 / \sqrt{\mathrm{m}}$, and would be greater than $10^{-9} \mathrm{~g} / \mathrm{VHz}$ for $\mathrm{m}<2 \mathrm{gm}$.[9]

Because the transducer measures coil velocity, at frequencies away from the resonance, the sensitivity degrades linearly with frequency. This poses a problem for seismology because many interesting signals occur at frequencies well below 1 $\mathrm{Hz}$, where geophone sensitivity is poor. One method for improving the low-frequency performance is to introduce a position transducer, and use the magnet/coil system to generate a rebalance force to hold the coil position constant. To resolve acceleration signals near $10^{-9} \mathrm{~g}$ at frequencies below $5 \mathrm{~Hz}$, the displacement transducer needs to resolve deflections of :

$$
\Delta \mathrm{Z}=\frac{\mathrm{A}}{\omega_{0}^{2}}=\frac{10^{-8} \mathrm{~m} / \mathrm{s}^{2}}{(2 \pi 5 \mathrm{~Hz})^{2}}=0.1 \AA .
$$

This is within the performance capabilities of the tunneling transducer, according to the data presented in Fig. 2 above. The tunneling transducer includes the advantage that the amplification and feedback circuitry can be implemented using discrete circuit elements, and does not need to be highly integrated in order to operate with the necessary performance.

Figure 7 shows a cross-section of a geophone which has been modified to include a tunneling position transducer. One electrode of the tunneling transducer is supported by the geophone case, and the other is attached to the moving coil. The position of the moving coil is controlled by passing a servo current through the coil. When there is an external acceleration, the servo force will balance the inertial force, thereby keeping the relative position of the coil constant.

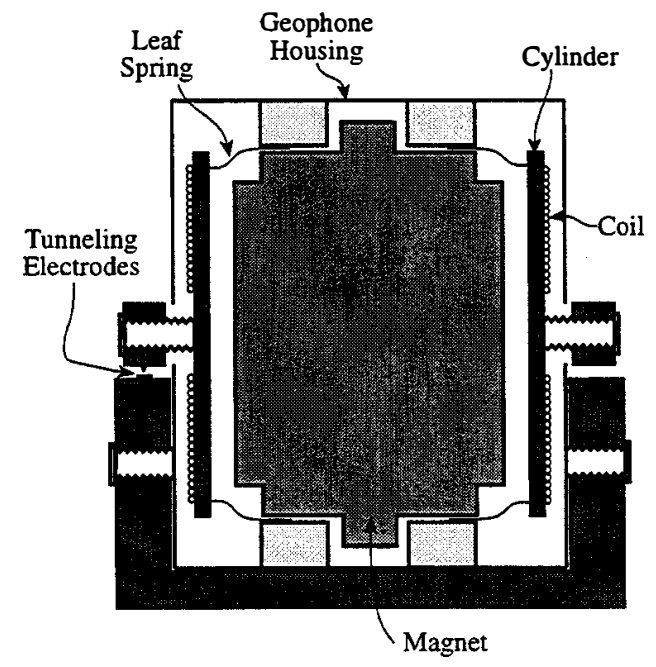

Figure 7. This drawing shows the internal structure of a geophone, and the modifications made to attach tunneling electrodes to the device.
As described elsewhere [7] there is no need for sharpened tips in a tunneling transducer. In this device, we have experimented with several geometries, including tunneling between the edges of a crossed pair of wires, between the end of a wire and a flat electrode, or between the side of a bent wire and a flat metal electrode. We have focused on these rather crude architectures because the low resonant frequency of the geophone will prevent the implementation of wide-bandwidth control.

During operation, motion of the tunnel tip leads to a change in tunnel current, which is converted to a voltage through a $10 \mathrm{M} \Omega$ resistor. An electronic control circuit (to be designed in the near future) generates a control voltage which is applied to the suspended coil in the geophone. If the control circuit is working properly, the inertial force on the coil $(\mathrm{F}=\mathrm{mA}=0.2 \mathrm{~N} / \mathrm{g}$ for a 20 gm mass) will be balanced by the electromagnetic force on the coil $(\mathrm{F}=0.02 \mathrm{~N} / \mathrm{V})$, so the sensitivity will be given by :

$$
S=\frac{\partial V}{\partial F} \frac{\partial F}{\partial A}=(50 \mathrm{~V} / \mathrm{N})(0.2 \mathrm{~N} / \mathrm{g})=10 \mathrm{~V} / \mathrm{g} \text {. }
$$

We have begun testing the prototype tunneling seismometer. We have encountered some difficulty implementing a controller which actually maintains a steady tunneling current because of the low resonant frequency of the proof mass One version of the control circuit operates with a large oscillation above $300 \mathrm{~Hz}$, in which the tunneling electrodes are alternately too far apart or in contact. Low frequency acceleration signals cause the controller to adjust the duty-cycle of this oscillation. By filtering the output, it is possible to detect low-frequency oscillations with this system. In a preliminary experiment, we confirmed the sensitivity of $10 \mathrm{~V} / \mathrm{g}$, and recorded the noise spectrum shown in Fig. 8. For frequencies below $15 \mathrm{~Hz}$, the resolution of this "almost"-tunneling geophone is already near $1 \mu \mathrm{g} / \mathrm{VHz}$, which is an excellent early result, and is competitive with good lab-grade accelerometers. With stable feedback control of the tunneling current, the fraction of the time that the sensor is tunneling can be increased dramatically, and we expect to achieve resolution approaching $10^{-9} \mathrm{~g} / \sqrt{\mathrm{Hz}}$. If successful, we plan to bring an operational tunneling geophone to our poster at the conference.

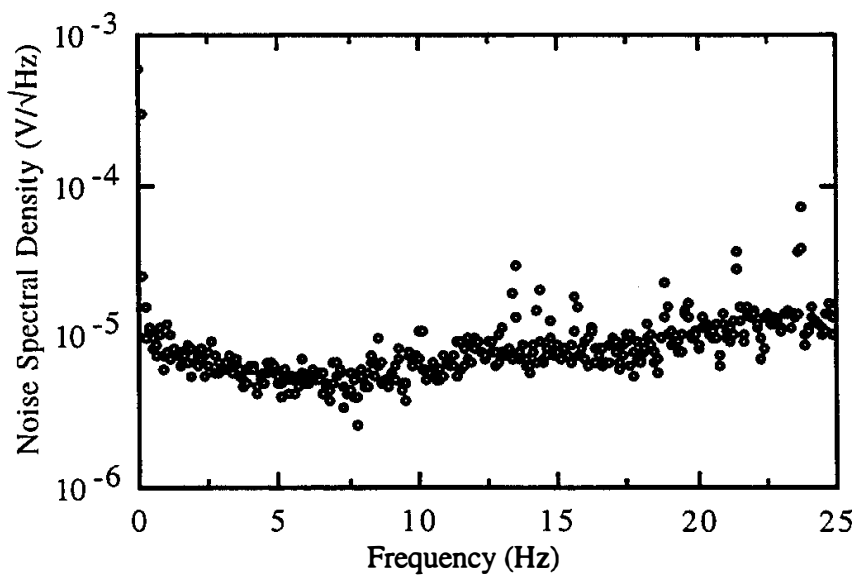

Figure 8 This graph shows the noise spectral density recorded at the output of the tunneling geophone. Since this device has a sensitivity of $10 \mathrm{~V} / \mathrm{g}$, the resolution is near $1 \mu \mathrm{g} / \sqrt{\mathrm{Hz}}$ across much of this low frequency range. 


\section{SURFACE MICROMACHINED TUNNELING TRANSDUCER}

To understand the sources of noise in tunneling devices, we have been interested in the development of test devices with structures other than the membrane device. One interesting approach is based on the work of Kobayashi and Fujita,[10] in which surface micromachining has been used to build a "Lateral Tunneling Unit". In their device, an electrostatic comb-drive is used to deflect a metal-coated polysilicon "finger" towards a fixed, metal-coated electrode.

We have used the MCNC MUMPS process for surface micromachined polysilicon structures, and have evaporated $\mathrm{Ti} / \mathrm{Pt} \mathrm{Au}$ metallization onto the structures after the HF-release step. This metal layer recipe provides good adhesion to the surface, an excellent diffusion barrier to prevent silicon diffusion through the gold, and a clean gold surface for the tunneling contacts. By evaporating after release, we rely on the overhanging polysilicon of each structural layer to provide shadow-mask isolation between the electrodes. Using this process, we are able to achieve the necessary clean metal electrodes, electrical isolation, and mechanical control that is necessary for the operation of a tunneling device. Figure 9 shows a drawing of the device design.

Difficulties encountered during early testing of this device have included stress in the evaporated metal layer which causes out-of-plane warp in the released structures. We recommend use of both structural polysilicon layers in the MUMPS process to stiffen the released structures, and careful minimization of the metal layer thickness. In our metal process, good metal electrodes can be achieved with as little as $80 \AA 80 \AA / 800 \AA$ of the $\mathrm{Ti} / \mathrm{Pt} / \mathrm{Au}$, respectively.

Preliminary testing of these devices is underway. Limitation of the out-of plane warp through the use of the 2-layer poly and minimization of the metal layer thickness has produced a device which acts as a reliable relay. Implementation of feedback control is underway, and we will present recommendations for

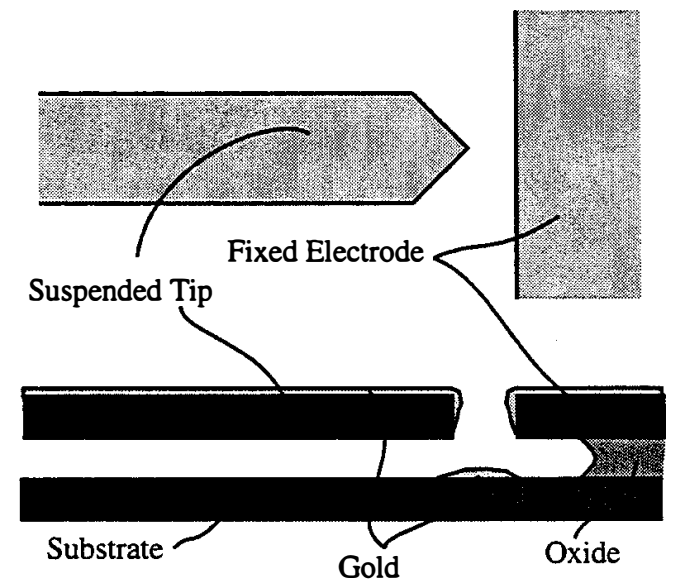

Figure 9 This drawing shows the method for obtaining tunneling electrodes from a comb drive-supported tip and a fixed electrode. The overhang which results from the release etch forms a natural shadow mask. The metals are evaporated with the sample mounted on a tilted planetary mount, so that the facing edges of the tunneling contact are partially coated. (After Kobayashi, et. al. [10]). feedback control in a subsequent publication. Once successful, a complete description of this device and its performance characteristics will be published as well. A significant advantage of fabrication of a test tunnel sensor structure through the MCNC process is that it will enable many new researchers to begin experimentation with tunneling devices.

\section{INTEGRATED TUNNEL CONTROLLER IN STANDARD CMOS}

In a previous publication, we have described a simple, discrete-component circuit which can be used to provide widebandwidth control for the membrane tunneling transducer. In this work, we report on work towards a single-chip control circuit for this device which can be made through MOSIS or the Stanford inhouse CMOS process.

While tunneling servo controllers can be quite simple to build from discrete components, these embodiments cannot generally be directly integrated. The tunneling current must be sensed and converted to a voltage. In discrete circuits this is accomplished with a large-value resistor (10M $\Omega$ ), which would use significant area in an integrated form. In addition, the common method to develop large resistances, well-implant snakes, would have prohibitively large leakage to substrate. Therefore we are developing a replacement for this component. We have constrained our search to implementations in common industrial CMOS, and assume that special purpose resistors and high quality bipolar devices are not available.

Many options are available for this application, including switched capacitor techniques, scaled current mirrors, and others. We have elected to convert the tunneling current to a voltage by dropping the current across a forward biased diode. Because of the logarithmic nature of the diode, this develops an easily measured voltage. Advantages of this approach include the small silicon area required by the diode and the high effective impedance of the diode at the small tunneling currents. At a current of a few nA, a diode will exhibit a bias of a few hundred millivolts, an equivalent (linearized) resistance on the order of a hundred megohms. This large equivalent resistance reduces the gain requirements of the amplifier stage. Also, the exponential dependence of tunneling current with tunneling gap displacement is linearized by the logarithmic diode, so displacement in the tunneling gap is roughly proportional to the developed voltage.

The forward current vs. voltage for a diode varies strongly from device to device and with temperature. Therefore, the control voltage must be generated and temperature compensated. The direct approach would be to drop a reference current across a reference diode identical to the sense diode and servo to the voltage across the sense diode to the voltage developed across the reference. While conceptually simple, developing the small well regulated reference current can be difficult in CMOS. Instead, we employ a method of scaling a voltage developed across matched diodes at larger currents.

The servo controller employs simple integral feedback with a bandwidth-limited op-amp topology. Since the electrostatic feedback of present tunneling transduces requires large drive voltages, we include a high voltage class $A$ output stage built with a high-voltage-FET transistor [11]. This transistor employs a lightly doped drain structure built from standard well implant to provide a high breakdown voltage. It can be fabricated in standard 
CMOS and provide over $100 \mathrm{~V}$ drive in our MOSIS parts and $60 \mathrm{~V}$ drive in our in-house CMOS.

The components of the circuit have been developed and tested and the circuit has been fabricated and is now under test.

\section{CONCLUSIONS}

In the past 2 years the pace of tunnel sensor development has quickened. Our research activities have been focused on the determination of the fundamental characteristics of tunneling transducers, and on the development of techniques to support commercialization. Recent low-frequency noise measurements have proven that the drift in tunnel sensors can be low enough to be dominated by thermal-mechanical effects in the supporting structure, and that the fundamental stability of these transducers is remarkably good. This work illustrates the importance of noise measurements for device performance characterization and points the way to performance improvements. We plan to continue and extend these measurements to include studies over wide temperature ranges and in the presence of vacuum and other controlled environments.

In parallel with the noise studies, we have begun development of a tunneling seismometer, and have presented some early, very encouraging results. In addition, we are developing a tunnel sensor fabrication process which can be carried out at MCNC. Finally, we are developing a single-chip, CMOScompatible tunnel sensor control circuit which can be obtained through MOSIS. Availability of these devices through foundries should further quicken the pace of tunnel sensor-based device and product development.

\section{ACKNOWLEDGMENTS}

We would like to thank W. Kaiser, T. George, S. Manion, C. Storment, and D. Marcus for discussions and support. N. Maluf and G. Kovacs have contributed directly to the integrated controller; the high-voltage circuitry was developed by Maluf. This work was supported by the Center for Space Microelectronics Technology, Jet Propulsion Laboratory, California Institute of Technology, and is sponsored by the National Aeronautics and Space Administration, Office of Space Access and Technology. We also acknowledge the National Science Foundation CAREER Award (ECS-9502046), the Charles Lee Powell Foundation, and the Terman Fellowship. We also thank The National Science Foundation for support of travel to this conference.

\section{REFERENCES}

[1] T.W. Kenny, J.K. Reynolds, J.A. Podosek, E.C. Vote, L.M. Miller, H,K, Rockstad, and W.J. Kaiser, "Micromachined Infrared Sensors using Tunneling Displacement Transducers,"Rev. Sci Instrum, 67 (1996), p. 112.

[2] H.K. Rockstad, J.K. Reynolds, T.K. Tang, T.W. Kenny, W.J. Kaiser, and T.B. Gabrielson, "A Miniature, High-Sensitivity, Electron Tunneling Accelerometer," Proceedings, Transducers '95, Vol. 2, (1995) p. 675.

[3] F.T. Hartley, B. Dolgin, and P.M. Zavracky, "Progress Towards a Bulk Micromachined Tunneling Tip
Microaccelerometer," Proceedings, Transducers '95, Late News Volume, (1995) p. 75.

[4] C. Yeh and K. Najafi, "A Low-Voltage Bulk-Silicon Tunneling-Based Accelerometer," to be published, (1995).

[5] C. Yeh and K. Najafi, "A Bulk-Silicon TUnneling-Based Pressure Sensor", Proceedings 1994 IEEE Solid State Sensor and Actuator Workshop, (1994) p. 123.

[6] L.M. Miller, J.A. Podosek, E. Kruglick, T.W. Kenny, J.A. Kovacich, and W.J. Kaiser, "A $\mu$-Magnetometer Based on Electron Tunneling," Proceedings 1996 IEEE-MEMS Workshop, (1996) p. 467 .

[7] T.W. Kenny, W.J. Kaiser, H.K. Rockstad, J.K. Reynolds, J.A. Podosek, and E.C. Vote, "Wide-Bandwidth Mechanical Elements for Tunneling Transducers," J. MicroElectroMechanical Systems 3, (1994) p. 97

[8] Mark Products L-28, Mark Products, 10502 Fallstone Road, Houston, Texas, 77099

[9] T.B. Gabrielson, "Mechanical-Thermal Noise in Micromachined Acoustic and Vibration Sensors," IEEE Electron Devices, 40, (1993) p. 903.

[10] D. Kobayashi, T. Hirano, T, Furuhata, and H. Fujita, "An Integrated Lateral Tunneling Unit," Proceedings 1992 IEEE MEMS Workshop, (1992) p. 214.

[11] N.I. Maluf, R.J. Reay, and G.T.A. Kovacs, "High-Voltage Devices and Circuits Fabricated using Foundry CMOS for use with Electrostatic MEM Actuators," Proceedings, Transducers '95, Vol. $1,(1995)$ p. 158. 